\title{
Analysis of Shaking-Induced Cherry Fruit Motion and Damage
}

\author{
Jianfeng Zhou ${ }^{1,2}$, Long He ${ }^{1}$, Manoj Karkee ${ }^{1,2}$, Qin Zhang ${ }^{1, *}$ \\ ${ }^{1}$ Center for Precision and Automated Agricultural Systems, Washington State University, \\ Prosser, WA 99350 USA \\ ${ }^{2}$ Department of Biological Systems Engineering, Washington State University, \\ Pullman, WA 99164 USA \\ "Corresponding author, (e-mail: qinzhang@ wsu.edu)
}

\begin{abstract}
\end{abstract}
High percentage of shaking-induced fruit damage is the major obstacle for mechanical harvesting of fresh-market sweet cherry. This study focused on the evaluation of the effect of vibration frequency on fruit motion, detaching time and damage during mechanical cherry harvesting with a shaker. A high-speed camera was used to capture the fruit trajectory on eighteen limbs excited at the frequencies of 10, 14 and $18 \mathrm{~Hz}$. Fruit detaching time and number of fruit-to-fruit and fruit-to-limb impact were extracted from the high speed videos for determining the factors affecting fruit removal and damage. Results showed that he majority of the four identified motion patterns were tilting and beam-column, accounting for more than $70 \%$ of all patterns. Fruit detaching time was significantly affected by vibration frequency with fast detaching at high frequencies. Vibration frequency affected the overall fruit damage percentage substantially, with the lowest damage percentage of $47.1 \%$ in $14 \mathrm{~Hz}$, which might be caused by high percentage of beam-column motion during vibration at the frequency. Meanwhile, $18 \mathrm{~Hz}$ vibration resulted in a higher percentage (35\%) of unmarketable fruits than that of $10 \mathrm{~Hz}$ and 14 Hz. On average, a fruit experienced 3.8 impacts against limbs and other fruit at $18 \mathrm{~Hz}$ excitation, substantially lower than that of 11.8 impacts at $14 \mathrm{~Hz}$ or 11.7 impacts at $10 \mathrm{~Hz}$ excitation. 
Overall, the results indicate that shorter duration of high-level mechanical impacts could induce higher fruit damage.

27 Keywords: Mechanical harvesting; sweet cherry; fruit damage; motion pattern; fruit detaching

\section{Introduction}

Most sweet cherries (Prunus avium L.) for fresh-market are harvested manually by migration pickers in the USA. Considering small fruit size and large number on a tree, cherry harvesting is labour intensive and time consuming. Due to shortage of skilled workers and increasing labour cost, harvest induced cost accounts for more than $50 \%$ of the annual production expenses for sweet cherry growers (Caplan, Tilt, Hoheisel, Baugher, 2014). Mechanical harvesting, especially vibrational harvesting, is a promising method that may improve the harvest efficiency and reduce harvest cost for tree fruit crops (Li, Lee, \& Hsu, 2011;

37 Zhou et al., 2013). However, previous research reports indicate that high percentage of harvestinduced fruit damage was observed in field trials, which is considered as a major obstacle for the acceptance of mechanical harvesting by the industries. For instance, Norton et al. (1962) and Halderson (1966) found considerable bruise damage on the harvested fruits in the field trials of mechanical harvesting for sweet cherries. Peterson, Whiting, and Wolford (2003) also reported a

42 damage percentage of $13.5 \%$ for "Van" sweet cherry fruits when they were harvested using an

43 impacting mechanical harvester. Recently, Chen et al. (2012) tried an impacting mechanical

44 harvester and a hand-held shaker in a sweet cherry orchard with fruit wall system, and reported 45 fruit damage rates of $27.4 \%$ and $23.9 \%$ respectively. High fruit damage restricted the adoption of 46 mechanical harvesting technologies in fresh market tree fruit crops. 
Mechanically harvested fruits usually experience two major processes, i.e. detaching

48 from limbs and catching with fruit catchers. Fruit damage may be caused by fruits hitting on tree

49 limbs or other fruits during vibration (detaching process) or hitting on the catching frame or

50 other fruits during catching process (Norton et al., 1962). The effect of mechanical impact on

51 fruit bruise damage due to fruit catching was reported by our research group (Zhou, He, Zhang,

52 Karkee, 2016) and was also explained in some other reports (Li \& Tomas, 2014; Opara \&

53 Pathare, 2014). In those researches, the determining factors of fruit damage were identified

54 mainly through impact tests or drop tests with laboratory setup. However, the issue of shaking-

55 induced fruit damage in detaching process has not been fully studied till now partly because of

56 the complicated responses of fruit-tree system to the vibration excitation (Li \& Thomas, 2014).

57 Stafford and Diener (1973) analysed the motion of apple fruits under vibrational excitation using

58 a shaker theoretically. They concluded that the shaking-induced fruit damage could be reduced

59 by limiting the shaking amplitude to minimize fruit displacement. Torregrosa, Albert, Aleixos,

60 Ortiz, and Blasco (2014) studied the motion of a citrus fruit due to vibrational excitation using a

61 slow-motion camera in laboratry condtion. The motion of the fruit was esimated using its

62 centroid, linear speed/acceleration and tilt angles. Zhou, He, Karkee, and Zhang (2013) tried to

63 identify three motion patterns and damage percentage of cherry fruits under vibrational

64 excitation in field condition for the first time, and the authors found that some motions were

65 more important than others in fruit detaching and damage. However, fruit motion of cherry due

66 to vibration still needs to be analysed clearly to understand the effect on fruit damage.

67

In practice, several elemental patterns, including pendulum, tilting, twisting and beam-

column motion (Fig. 1), or their combinations have been widely used to simplify the analysis of

69 fruit motion under vibrational excitation. Diener, Mohsenin, and Jenks (1965) confirmed the 
presence of the first three basic motion patterns in vibrational harvesting of apples. Rumsey and

71 Barnes (1970) found similar motion patterns in oranges and grapefruit as apples under

72 vibrational excitation. Such motion patterns resulted in different fruit detaching forces; a

73 pendulum motion exerted tensile force along the stem, tilting motion caused bending stress,

74 twisting motion generated torsional stress, and the beam-column motion resulted in large tensile

75 force because of the sudden change in the direction of motion (Cooke \& Rand, 1969; Rand \&

76 Cooke, 1970). It was also found that stem fatigue during a repeated bending motion played a

77 more important role in fruit detachment than the tensile force (Cooke \& Rand, 1969; Lamouria \&

78

79

80

8

Brewer, 1965; Rand \& Cooke, 1970). (a)

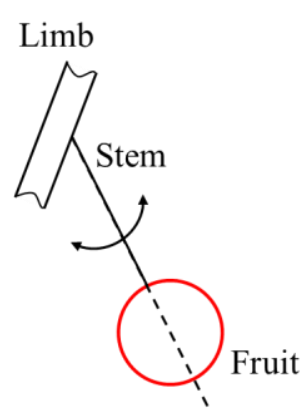

(b)

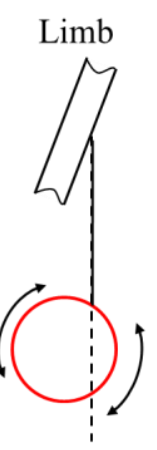

(c)

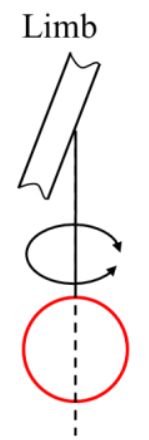

(d)

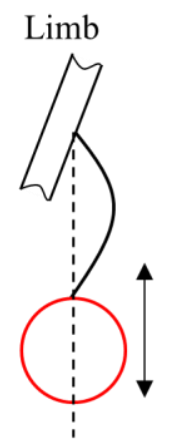

Fig. 1 - Elemental motion patterns of fruit under vibrational excitation. (a) Pendulum, (b) Tilting, (c) Twisting and (d) Beam-Column

Fruits like apples and oranges are larger in size ('big') and usually grow separately from each other, while 'smaller' fruits like cherries usually grow in clusters with 10 or more fruits in a cluster. When fruits in a cluster are excited using a shaker during mechanical harvesting, it is easy to induce fruit-to-fruit and fruit-to-limb impacts, which may result in fruit damage and fruit trajectories unpredictable. To reduce the shaking-induced fruit damage in sweet cherries, it is important to determine the major factors inducing such damage. The goal of this research was to 
92 identify the possible causes of shaking-induced fruit damage in detaching process in fruit scale.

93 To achieve this goal, three specific research objectives were: (1) to identify the motion patterns

94 of fruits excited at different frequencies; (2) to determine factors affecting fruit detaching time;

95 and (3) to determine factors causing fruit damage during fruit detaching.

96

\section{2. Materials and methods}

$98 \quad 2.1$ Experiment setup harvesting season at the first week of July, 2013. Test trees were trained to a fruiting wall system with Y-trellised canopy architecture. In total 18 limbs with similar diameter were chosen from randomly selected 18 trees in the orchard. One or two fruit clusters, including 10-20 fruits, were carefully selected as sample groups from the 18 limbs by avoiding those with visible fruit defects

105 (dents, holes or bruise). All fruits in a group were labelled for tracking their quality and motion in further analysis. Prior to test, 240 randomly selected fruits from the selected limbs were pulled

107 using a digital force gauge (DS2-4, Imada, Northbrook, IL, USA) with a custom fitted polyvinyl 108 chloride attachment (Chen et al., 2012). The force gauge measured and recorded the peak 109 retention force for detaching a fruit from trees (i.e. pedicel-fruit retention force, PFRF). The 110 detached fruits were collected in paper bags and sent to laboratory for measuring firmness using

111 a firmness tester (Firm Tech 2, Bio Works, Wamego, Kansas), and weighing using a digital 112 balance. Meanwhile, the maturity level of those fruits was also estimated using fruit skin colour 113 (Kappel, Fisher-Fleming, \& Hogue, 1996) graded with a cherry colour chart (Cerise, Centre 
114 Technique Interprofessionel des Fruit et Légumes, Paris, France). Some of the general

115 parameters of the orchard and trees are reported in Table 1.

Table 1. Tree and fruit parameters of test orchard

\begin{tabular}{ccccccc}
\hline Variety & $\begin{array}{c}\text { Age } \\
\text { (years) }\end{array}$ & Rootstock & Maturity & $\begin{array}{c}\text { Fruit } \\
\text { weight }(g)\end{array}$ & PFRF $(N)$ & $\begin{array}{c}\text { Firmness } \\
(\mathrm{g} / \mathrm{mm})\end{array}$ \\
\hline Skeena & 10 & Gi12 & $6.3 \pm 0.2$ & $10.4 \pm 1.6$ & $5.6 \pm 1.4$ & $308.6 \pm 49.8$ \\
\hline
\end{tabular}

A hand-held electric limb shaker was used to apply vibrational excitation to selected tree

121 limbs. The shaker was adapted from an electronic reciprocating saw (model: 6519, Milwaukee

122 Electric Tool, Brookfield, WI, USA) by replacing the blade with a custom fitted hook. The

123 shaker provided a fixed stroke of $32.0 \mathrm{~mm}$ with adjustable frequency up to $46.0 \mathrm{~Hz}$. The

124 vibration frequency was regulated using a motor controller modified from an off-the-shelf motor

125 driver (SyRen 10, Robotshop, Swanton, VT, USA). Prior to each treatment, the vibration

126 frequencies of the shaker were pre-set using a laser tachometer (HHT12, OMEGA Engineering,

127 Stamford, CT, USA). To harvest fruits, the shaker hooked to a selected limb at the position

128 (shaking position) of approximate $0.3 \mathrm{~m}$ below the target cherry clusters (Fig. 3a). The diameter

129 the limb at the shaking position was measured with a vernier calliper. To avoid damage induced

130 by catching frames, detached fruits were caught using a soft cloth sheet laid approximately 0.05

$131 \mathrm{~m}$ underneath the fruit clusters (above the shaker).

A high-speed camera (HiSpec 1, Fastec Imaging, San Diego, CA, USA) was used to

133 record the motion of sample fruits excited by different vibration frequencies. Fig. 2a shows the

134 field setup of the camera and the relative relationship between the camera and tentative shaking

135 plane and fruit motion plane. From the figure one can see that a limb was excited by the shaker

136 horizontally in the plane perpendicular to the camera axis (i.e. the shaking plane). The attached 
137 fruits might swing randomly in all directions because of the impact by other fruits or limb during 138 vibration. The tentative field of view of the camera was set to cover the motion range of sample 139 fruits by adjusting the distance between the camera and fruits (Fig. 2b). The camera's frame rate 140 was set at 700 frames per second (fps) (i.e. $1 / 700 s=1.4 \mathrm{~ms}$ ) which was 38.8 times faster than 141 the maximum vibration frequency of $18 \mathrm{~Hz}$ used in this study. To gain a clear visibility of the 142 target fruit samples in the images, the neighbour fruits and leaves were removed before test.

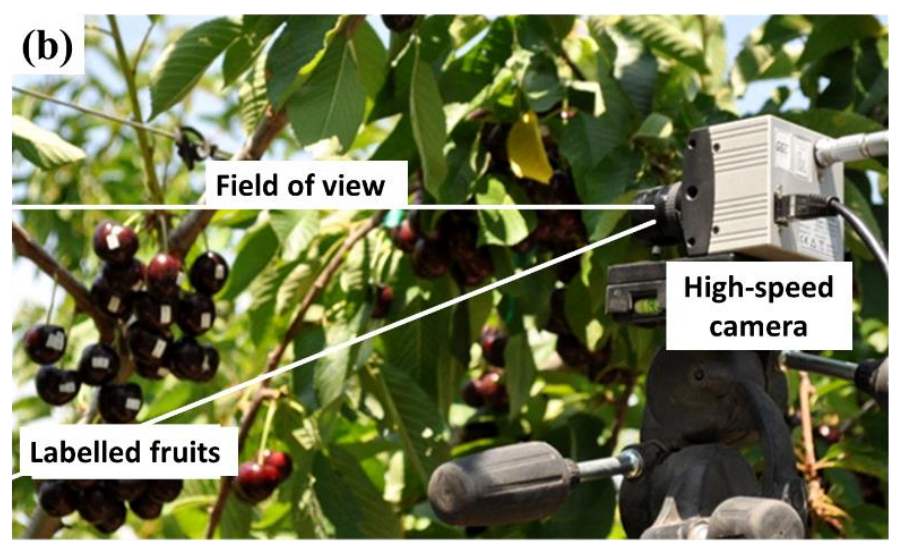

Fig. 2 - Experimental setup. (a) Illustration of the experimental setup. A camera was used to record the motion of fruits excited with a limb shaker. The tentative shaking plane was perpendicular to the camera axis; (b) Field setup. The camera's field of view covered all the labelled fruits (sample groups).

The effect of vibration frequency on fruit motion and damage was investigated on 18 selected limbs excited by three frequencies of 10, 14 and $18 \mathrm{~Hz}$, six limbs (replicates) for each frequency. A total of 247 fruits from 18 limbs were harvested from trees. There are three potential detachment conditions in the harvested fruits, with stem, without stem and broken stem (with part of stem). The number of fruits with or without stems in each group were counted and the percentage of fruit with stem was calculated using the ratio of number of fruits with stem to the total number of fruits harvested at each frequency. The harvested fruits were carefully collected and sent to laboratory right after experiment. Their skin colour and weight was 
measured in laboratory and then all samples were stored in a cold storage room $\left(\sim 0^{\circ} \mathrm{C}\right.$ with $>95 \%$ $\mathrm{RH}$, forced-air). Meantime, 180 defect-free fruits were manually picked from the selected trees as reference to compare the quality of mechanical harvested fruits with manually harvested fruits. All fruit samples were carefully collected into paper bags and also stored in the same cold storage room for further quality assessment.

\subsection{Fruit motion pattern and detaching time}

Motion trajectories of cherry fruits under vibrational excitation are hit by other fruits in a cluster and limbs, which makes their trajectory difficult to be analysed. In each vibration cycle (i.e. one stroke), more than one motion patterns may be observed (Stafford \& Diener, 1973). To tentatively extract fruit trajectory from the recorded high-speed videos, the centroids of fruits were identified and traced using Matlab 2014a (The MathWorks, Natick, MA, USA). However, it was found that the trajectories of fruits were not always in the same motion plane. Therefore, it is difficult to obtain the accurate fruit trajectories using a 2D imaging method. In this paper, we reported two examples of fruits' approximate trajectories by assuming the fruits were moving in the same plane to show the complexity.

The basic motion patterns of each fruit during detach process were identified by palying the high-speed videos frame-by-frame. The unique label of each fruit was used as a marker to track their motion. Some fruits might be hidden by other fruits or tree limbs during vibration and made it difficult to recognise their labels. However, usually part of fruits could be seen and they can be traced back when labels showed in the images. Additionally, the hidding time of a fruit was usually very short (less than two cycles). The error casued by the overlapping of fruits was neglected in this study. The fruit motion pattern was recognized visually according to the 
181 classification in Fig. 1, and the number of each motion pattern was recorded. Meanwhile, time

182 used to detach a fruit, i.e. fruit detaching time, was recorded using the time marker in the high-

183 speed videos. Fruit detaching time was used to describe the fruit harvest efficiency. Furthermore,

184 the vibration cycles used to detach a fruit (detaching cycles) were also calculated using the

185 production of fruit detaching time and vibration frequency.

186

$187 \quad 2.3$ Fruit damage analysis

188 Damaged fruits were graded into four levels using 'USDA Standards for Sweet Cherry

189 Grades' (USDA-AMS, 1997) and the 'Shipping Point and Market Inspection Instructions'

190 (USDA-AMS, 2005). Level-1 damage was defined as one or more small but negligible dents on

191 fruit surface. Fruits with level-1 were still marketable. Level-2 damage was defined as small

192 dents with less than 6.4-mm diameter on fruit surface making those fruits unmarketable. If the

193 damage region was larger than $6.4 \mathrm{~mm}$ in diameter, it was defined as level-3 damage, and level-4

194 when the damaged area exceeded $9.5 \mathrm{~mm}$ in diameter. Fig. 3 presents a few fruits in different

195 levels of damage. Fruit damage was assessed after one week of cold storage $\left(\sim 0^{\circ} \mathrm{C}\right.$ with $>95 \%$

$196 \mathrm{RH})$, when the damage region would develop and become softer than the area without impact

197 and the skin turn wrinkled (Thompson, Grant, Kupferman, Knutson, 1997). This allows the easy

198 identification of the bruise region on the fruit by touching the surface with a finger and visually

199 checking the skin. A crystal plastic card with two holes in diameters of $6.4 \mathrm{~mm}$ and $9.5 \mathrm{~mm}$ was

200 used to grade the damage region into four levels.

201 


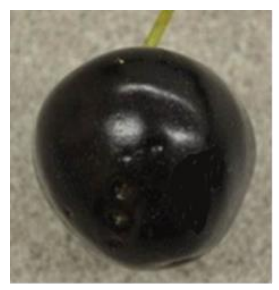

(a)

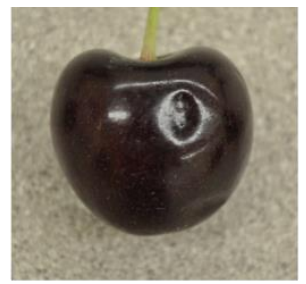

(b)

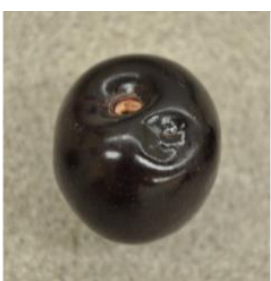

(c)

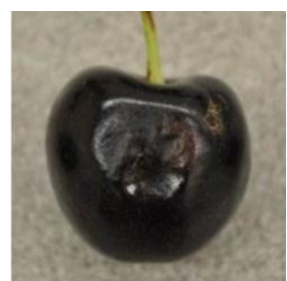

(d)

Fig. 3 - Illustration of different levels of fruit damage; (a) Level-1 damage, fruit with small dents, still marketable; (b) Level-2 damage, unmarketable pitting damage; (c) Level-3 damage, diameter of damage region was bigger than $6.4 \mathrm{~mm}$; and (d) Level-4 damage, diameter of damage region was bigger than $9.5 \mathrm{~mm}$. Fruits in damage more than level-2 were not marketable based on USDA standard.

The effect of vibration frequency on the fruit damage was evaluated using the factor of fruit damage percentage of the overall four levels and the individual level. Overall Fruit damage percentage was defined as the ratio of the number of damaged fruits to the total number of fruits in a sample group (target fruits on a limb) using the following equation.

$$
P_{d}=\frac{N_{d}}{N_{t}} \times 100
$$

where, $P_{d}$ was the fruit damage percentage $(\%), N_{d}$ was number of damaged fruits in a group.

Meanwhile, to evaluate the damage in different time period, a partial fruit damage percentage was also defined as the ratio of the number of damaged fruits to the number of fruits harvested in five vibration cycles from a limb.

The collected data of fruit weight, maturity level and fruit damage percentage were analysed using software SigmaPlot (ver. 11.0, Systat Software, San Jose, CA, USA). The significance of differences in the average values of those data was compared using one way ANOVA (Tukey test) function in SigmaPlot at 0.05 level.

\section{Results and discussion}

\subsection{Fruit motion pattern}


The approximate trajectories of fruits under vibrational excitation were extracted using

high speed videos with the assumption that they were moving in the same plane. The centroid trajectories of two fruits excited at $10 \mathrm{~Hz}$ were plotted in Fig. 4. It can be seen that in the first excitation period (marked with \#1), both fruits were accelerated and swung in the direction of shaking. Similarly, the fruit trajectory in \#2 excitation period showed as pendulum motion around the stem-limb junction as expected. However, their trajectories were substantially different in the following time periods and did not showed regular patterns. Their trajectories might be affected by the impacts from other fruits and limbs which were unpredictable. The figure also shows that Fruit \#1 (Fig. 4a) in the outer layer of fruit cluster might swing higher (in larger amplitude) compared to fruit \# 9 (Fig. 4b) in inner layer of the cluster. The motion path in the inner layer was altered more often due to more chances hit by other fruits. Quantitative analysis on fruit trajectory may be an interesting project for future research.
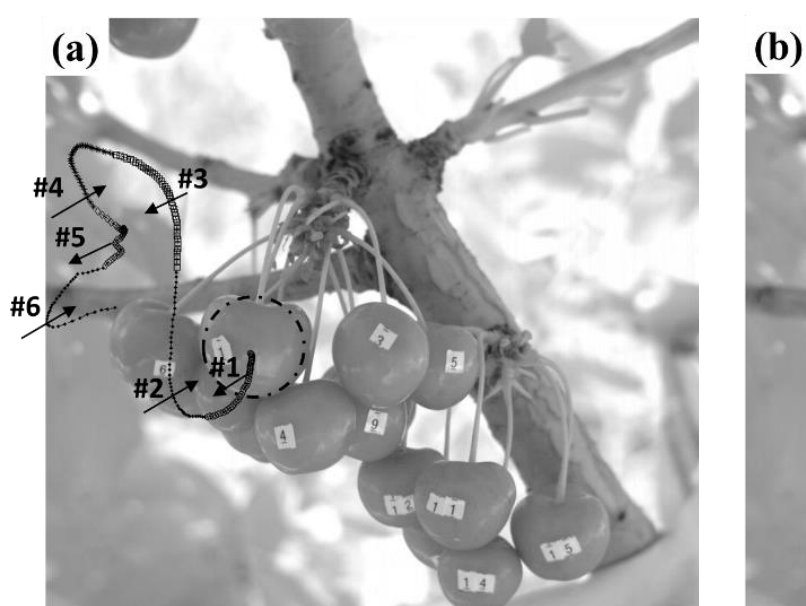

(b)

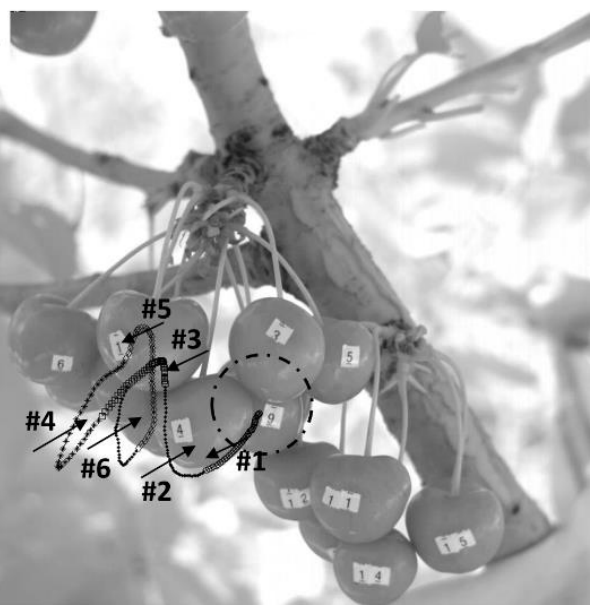

Fig. 4 - Approximate motion trajectory of: (a) fruit 1 and (b) fruit 9 from the same fruit cluster on a limb excited at $10 \mathrm{~Hz}$. Numbers (\#1 - \#6) indicate the motion sequence of fruit centroid and arrows indicate the moving direction of the limb at the corresponding time period.

The number of each motion pattern of the 247 fruits at three vibration frequencies of 10 ,

24414 and $18 \mathrm{~Hz}$ was counted using the high-speed videos. The average number and the percentage 
245 of each pattern under three vibration frequencies are shown in Fig. 5. It can be seen from Fig. 5a

246 that the accumulated numbers of four motion patterns used to remove a fruit at vibration

247 frequency of $10 \mathrm{~Hz}$ were approximately three times more than those at $18 \mathrm{~Hz}$. For $10 \mathrm{~Hz}$

248 vibration, it took 24.9 accumulated motion patterns in average (ranged from 3 to 57), consisting

249 of 1.1, 6.3, 9.0 and 8.5 twisting, pendulum, tilting and beam-column motion patterns,

250 respectively. In comparison, the corresponding number of motion patterns reduced to 18.6

251 (ranged from 4 to 48 , consisted of 1.7, 3.3, 5.5, and 8.1 twisting, pendulum, tilting and beam-

252 column motion patterns) and 8.8 (ranged from 3 to 30, consisted of 1.0, 0.8, 4.1, and 2.9 twisting,

253 pendulum, tilting and beam-column motion patterns) when vibration frequency increased to14

254 and $18 \mathrm{~Hz}$, respectively. The results also indicate that there were substantially more tilting and

255 beam-column motions than pendulum and twisting motions. From Fig. $5 \mathrm{~b}$ a trend can be found

256 that accumulated number of tilting and beam-column motion accounts for $70.2 \%, 73 \%$ and $80 \%$

257 of all the motion patterns at 10,14 and $18 \mathrm{~Hz}$, indicating the importance of these two motion

258 patterns in fruit removal during cherry harvesting. As previous discussion that tilting motion

259 might cause bending stress and beam-column motion might result in large tensile force, therefore,

260 most of fruits might be detached by the multiple times of bending and beam-column motions. 
(a)

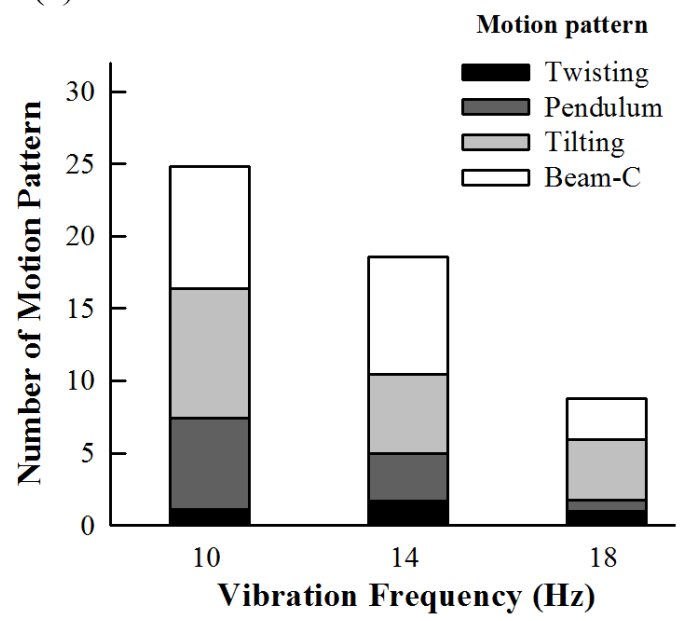

(b)

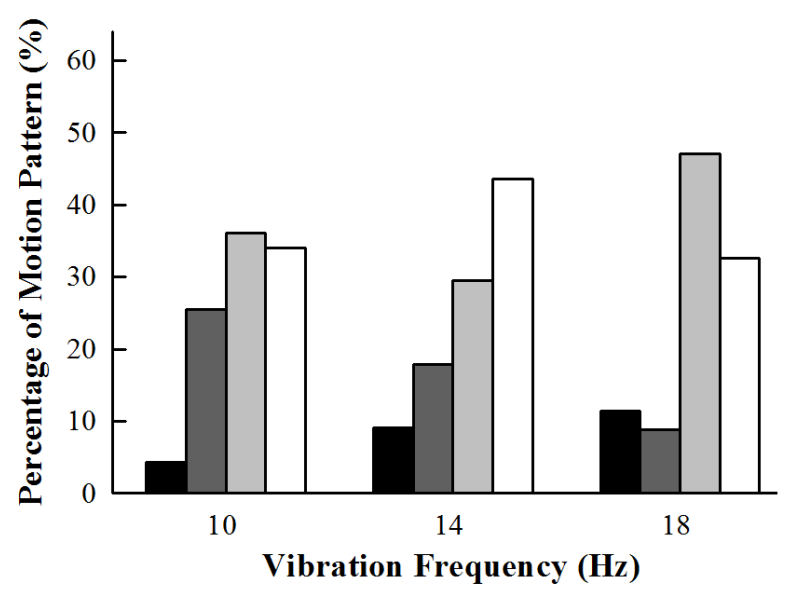

Fig. 5 - Average number and percentage of motion patterns of twisting, pendulum, tilting and beam-column in fruit detaching process; (a) Average number of each motion pattern used to detach a fruit at three vibration frequencies; (b) Percentage of each motion pattern in the detachment of a fruit at three vibration frequencies.

The percentages of fruits with stem at three vibration frequencies are shown in Table 2, which shows that $10 \mathrm{~Hz}$ vibration resulted in less fruits with stem. The detachment condition might be affected by motion patterns. As discussed previously, tilting motion may result in flexural stress at the pedicel-fruit abscission zone and the twisting motion can result in a shear stress or torsional stress in the abscission zone. Both motion patterns might have higher potential to detach fruits without stem than other motions. According to Fig. 4a, fruits at $10 \mathrm{~Hz}$ experienced more times of both motion (1.1 times of twisting and 8.5 times of tilting) comparing to $14 \mathrm{~Hz}$ (1.7 and 5.5 times respectively) and $18 \mathrm{~Hz}$ (1.0 and 4.1 times respectively), which might result in more fruits without stem.

\section{Table 2 - Percentage of fruits with stem at three vibration frequencies}

\begin{tabular}{cccc} 
Frequency & $10 \mathrm{~Hz}$ & $14 \mathrm{~Hz}$ & $18 \mathrm{~Hz}$ \\
\hline Fruit with stem $(\%)$ & 5.9 & 10.8 & 9.4 \\
\hline
\end{tabular}

\subsection{Fruit detaching time}


Table 3 lists the limb diameters at shaking position, average maturity level and average

282

283

284

285

286

287

288

289

290

291

292

293

294

295

296

297

298

299

300

301

302

weight of harvested fruits, as well as fruit detaching time and cycles of each group. It can be seen

that the limb diameter at the shaking positon was very consistent, except group 7 and 13.

However, there was no a strong correlation between the limb diameters with fruit detaching time.

In terms of fruit maturity level and fruit weight, although they might be substantially different

from tree to tree, there was no significant difference among three treatments (frequencies).

Therefore, it is suitable to compare the effect of vibration frequency on fruit motion and damage

between different treatments. As expected, it can be seen from Table 3 that the both average

values of fruit detaching time and fruit detaching cycles at lower vibration frequency were

significantly longer than those at higher frequencies, with $2.7,1.5$ and $0.5 \mathrm{~s}$ for detaching time

and 20.7, 20.6 and 8.5 for detaching cycles at 10,14 and $18 \mathrm{~Hz}$, respectively. This result

indicates that higher vibration frequency could obtain higher harvest efficiency, which agrees

with previous reports (Castro-Garcia et al., 2009; Zhou et al., 2013). However, fruit detaching

time was not consistent from tree to tree, which might because that the capacity of limbs to

transmit kinetic energy from the shaker to fruits was different (Du, Chen, Zhang, Scharf, Whiting,

2012; He et al., 2013; Horvath \& Sitkei, 2001). The factors affecting energy transmission may be

determined by the combined effect of properties of trees (structure, size, diameter, etc.), fruits

(weight, maturity, stem length, etc.), and vibration technologies (excitation frequency, amplitude,

shaker mass, position, etc.).

Table 3 - Diameter of shaking position, average maturity level, weight and cycles used to detach a fruit in each frequency treatment.

\begin{tabular}{ccccccc}
\hline Group & $\begin{array}{c}\text { Frequency } \\
(\mathrm{Hz})\end{array}$ & $\begin{array}{c}\text { Diameter } \\
(\mathrm{mm})\end{array}$ & Maturity & Weight $(\mathrm{g})$ & $\begin{array}{c}\text { Detaching } \\
\text { time }(\mathrm{s})\end{array}$ & $\begin{array}{c}\text { Detaching } \\
\text { cycles }\end{array}$ \\
\hline 1 & 10 & 113.0 & $6.3 \pm 0.3$ & $10.8 \pm 1.6$ & $1.7 \pm 0.9$ & $17.1 \pm 8.8$ \\
2 & 10 & 102.0 & $6.4 \pm 0.2$ & $10.2 \pm 0.9$ & $2.9 \pm 0.2$ & $28.8 \pm 4.3$ \\
3 & 10 & 128.0 & $6.1 \pm 0.2$ & $10.1 \pm 1.2$ & $5.6 \pm 0.6$ & $56.4 \pm 6.4$
\end{tabular}




\begin{tabular}{ccccccc}
4 & 10 & 141.0 & $6.2 \pm 0.3$ & $11.3 \pm 0.9$ & $4.3 \pm 0.7$ & $43.4 \pm 7.4$ \\
5 & 10 & 108.0 & $6.3 \pm 0.2$ & $12.3 \pm 1.1$ & $0.6 \pm 0.1$ & $5.7 \pm 1.2$ \\
6 & 10 & 118.0 & $6.2 \pm 0.2$ & $9.4 \pm 1.0$ & $1.0 \pm 0.4$ & $10.3 \pm 4.3$ \\
Mean & & 118.3 & $6.3 \pm 0.2^{\mathrm{a}}$ & $10.7 \pm 1.4^{\mathrm{a}}$ & $2.7 \pm 1.6^{\mathrm{a}}$ & $27.0 \pm 16.4^{\mathrm{a}}$ \\
\hline 7 & 14 & 99.0 & $6.2 \pm 0.2$ & $8.1 \pm 0.7$ & $1.5 \pm 0.7$ & $21.7 \pm 10.0$ \\
8 & 14 & 122.0 & $6.1 \pm 0.1$ & $11.3 \pm 0.7$ & $1.5 \pm 0.6$ & $19.5 \pm 12.0$ \\
9 & 14 & 121.0 & $6.1 \pm 0.1$ & $10.5 \pm 1.2$ & $1.4 \pm 0.8$ & $26.8 \pm 9.3$ \\
10 & 14 & 134.0 & $6.2 \pm 0.2$ & $10.7 \pm 1.5$ & $1.9 \pm 0.6$ & $24.2 \pm 9.3$ \\
11 & 14 & 139.0 & $6.2 \pm 0.0$ & $10.7 \pm 1.1$ & $1.2 \pm 0.5$ & $10.3 \pm 7.6$ \\
12 & 14 & 128.0 & $6.2 \pm 0.1$ & $10.4 \pm 1.6$ & $0.7 \pm 0.5$ & $21.1 \pm 9.7$ \\
Mean & & 123.8 & $6.2 \pm 0.1^{\mathrm{a}}$ & $10.3 \pm 1.4^{\mathrm{a}}$ & $1.5 \pm 0.7^{\mathrm{b}}$ & $20.6 \pm 10.9^{\mathrm{b}}$ \\
13 & 18 & 146.0 & $6.2 \pm 0.1$ & $11.1 \pm 1.9$ & $0.4 \pm 0.1$ & $22.7 \pm 14.7$ \\
14 & 18 & 109.0 & $6.4 \pm 0.2$ & $12.6 \pm 0.9$ & $0.2 \pm 0.0$ & $10.3 \pm 1.3$ \\
15 & 18 & 110.0 & $6.2 \pm 0.1$ & $10.6 \pm 1.2$ & $0.2 \pm 0.1$ & $6.4 \pm 2.8$ \\
16 & 18 & 128.0 & $6.6 \pm 0.3$ & $9.3 \pm 1.1$ & $0.2 \pm 0.0$ & $4.0 \pm 0.8$ \\
17 & 18 & 129.0 & $6.1 \pm 0.1$ & $9.9 \pm 0.7$ & $1.3 \pm 0.8$ & $4.1 \pm 1.3$ \\
18 & 18 & 122.0 & $5.8 \pm 0.1$ & $12.2 \pm 1.1$ & $0.4 \pm 0.1$ & $3.5 \pm 0.6$ \\
Mean & & 124.0 & $6.2 \pm 0.3^{\mathrm{a}}$ & $10.9 \pm 1.7^{\mathrm{a}}$ & $0.5 \pm 0.4^{\mathrm{c}}$ & $8.5 \pm 8.8^{\mathrm{b}}$ \\
\hline
\end{tabular}

Numbers with same characters indicate no significant difference.

Additionally, one factor we observed that might affect fruit detaching was trellis wire used to train tree canopy. To compare the effect, two example groups from $10 \mathrm{~Hz}$ treatment were group 3 with the average detaching time of $5.6 \mathrm{~s}$ and group 5 with the average detaching time of 0.6 s. Fig. 6 shows the different fruit locations of both groups; fruits in group 3 (Fig. 6a) were near a trellis wire and fruits on group 5 were free from the strain of trellis wires (Fig. 6b). It was observed that the vibration amplitude of the limb in group 3 was substantially smaller than that in group 5. Large vibration amplitude generated high vibration energy to remove fruit quickly.

Therefore, trellis wire might have adverse effect on the fruit detaching. 
(a)

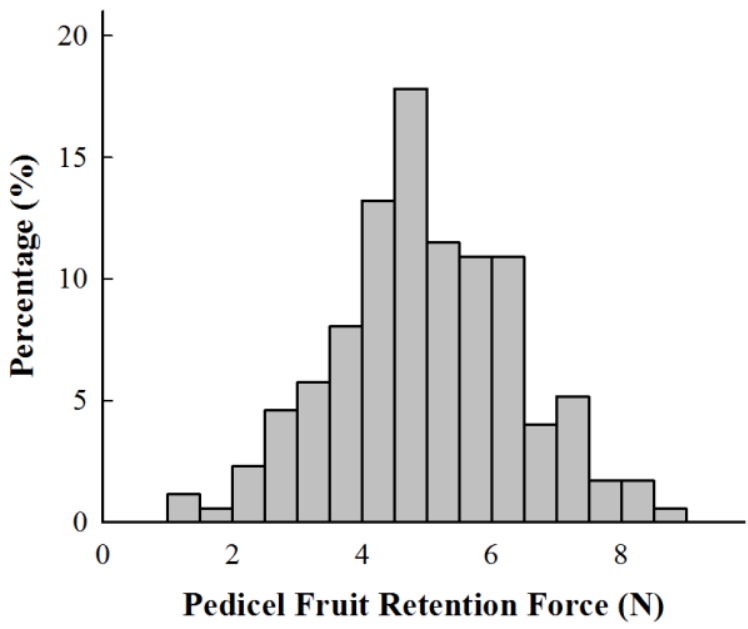

(b)

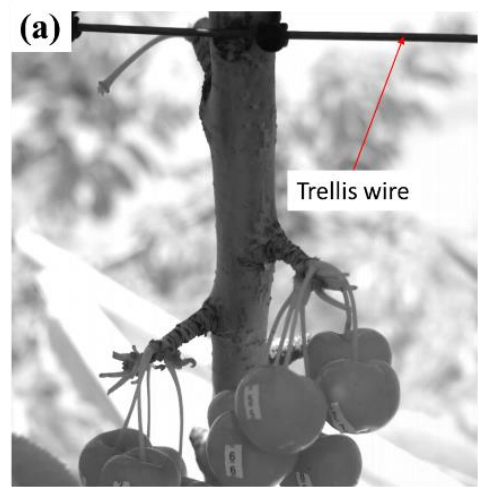

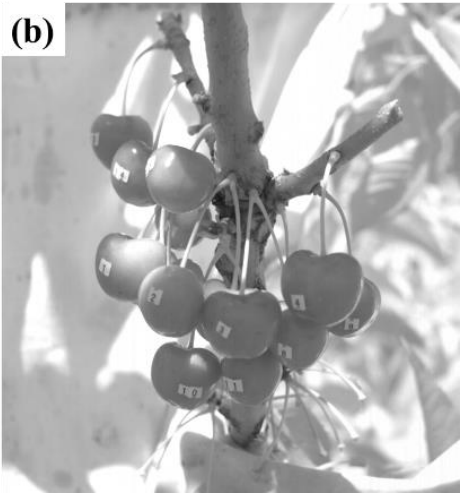

Fig. 6 - Trellis wire influenced fruit detaching time: (a) fruit zone near a wire (averaged $5.6 \mathrm{~s})$; (b) fruit zone away from trellis wire (averaged $0.5 \mathrm{~s}$ ).

Another factor related to fruit detaching may be pedicel fruit retention force (PFRF). It is considered as one of the most important factors determining fruit removal (Peterson, Whiting, Wolford, 2003). The PFRF of 240 fruit samples from eight trees in the test orchard was summarized in Fig. 7. One may see from Fig. 7a that the majority (64\%) of testing fruits had a PFRF of 4.0-6.5 N, 22\% of the fruits had a PFRF of 1.2-4.0 $\mathrm{N}$ and $14 \%$ in a $6.5-8.6 \mathrm{~N}$ range. Fig. $7 \mathrm{~b}$ also shows that the PFRF from one tree might be significantly different from other trees, which might be one of the reasons that the fruit detaching time varied tree to tree.

(b)

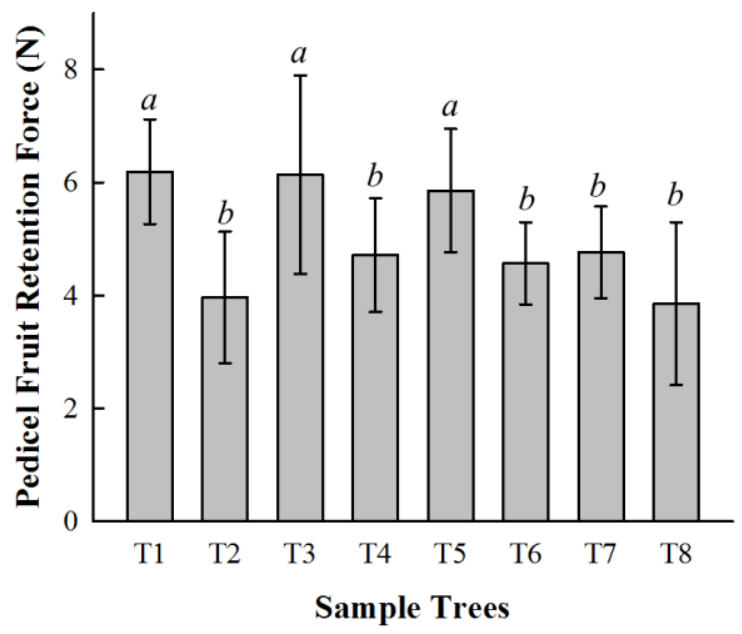


327

328

329

330

331

Fig. 7 - Pedicel fruit retention force; (a) Overall histogram of PFRF of fruit samples from eight trees; (b) PFRF of fruits from each selected trees with different letters indicating significant difference.

\subsection{Shaking induced fruit damage}

Shaking induced fruit damage in fruit detaching process was mainly the results of mechanical impacts in forms of fruit-to-fruit and fruit-to-limb impact during vibration. The damage analysis was based on the 247 fruit harvested from the 18 selected limbs. Fig. 8a presents changing trend of the overall fruit damage percentage accumulated with the increase of vibration cycles (Fig. 8a). The overall fruit damage percentage continued raising up from 25.3\%, $7.4 \%$ and $54.9 \%$ at the first 5 -cycle to $55.2 \%, 47.1 \%$ and $63.4 \%$ in the last cycle at the vibration frequency of 10,14 and $18 \mathrm{~Hz}$, respectively. The results show that the overall fruit damage percentage of $14 \mathrm{~Hz}$ was always lower than that of both 10 and $18 \mathrm{~Hz}$, which was also confirmed by other researches (Zhou, He, Zhang, Karkee, 2014). The lower fruit damage might be a reason of different motion patterns due to vibration frequency. It can be seen from Fig. 5 that the percentage of beam-column motion pattern at $14 \mathrm{~Hz}$ vibration was higher than that of $10 \mathrm{~Hz}$.

Beam-column motion pattern may be a safer trajectory since it only generates axial motion along stem and has less chance to hit other fruits or limbs. 


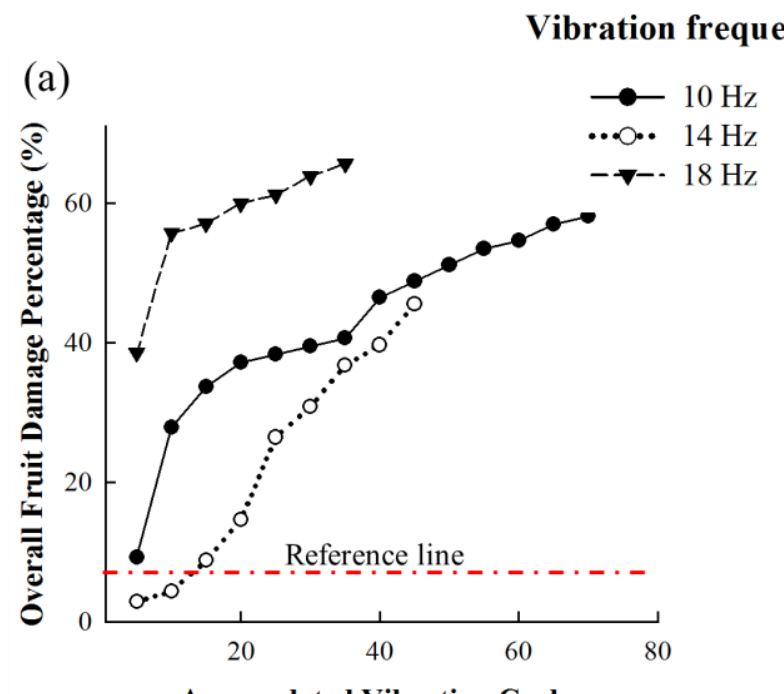

Accumulated Vibration Cycles

(b)

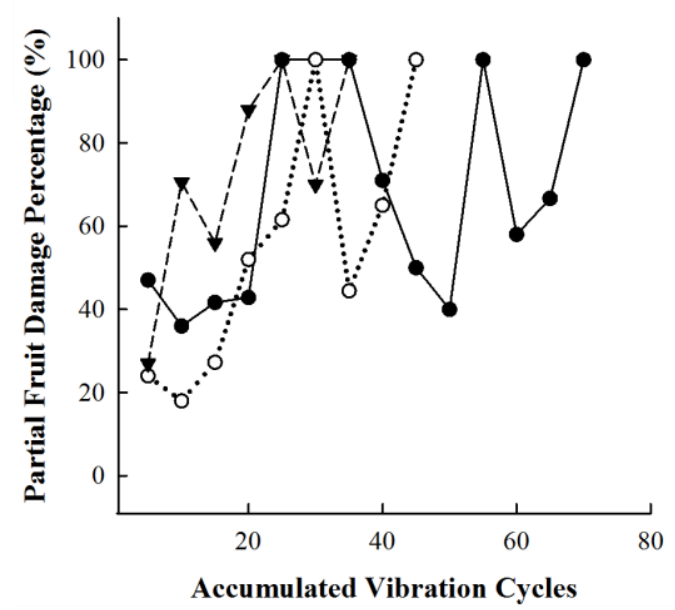

Fig. 8 - Fruit damage percentage at three vibration frequencies. (a) Overall fruit damage percentage over the accumulated vibration cycles (vibration time $\times$ frequency). Red dash dot line indicates the damage percentage $\mathbf{7 . 5 \%}$ ) of manually harvested fruits as a reference, including $6.7 \%$ damaged fruits in Level-2 and $0.8 \%$ fruits in Level-3. (b) Partial fruit damage percentage within a short interval of five vibration cycles (vibration time $\times$ frequency).

Fig. $8 \mathrm{~b}$ shows changing trend of the partial fruit damage percentage at each 5-cycle

interval. In general, fruits detached within less vibration cycles had less damage comparing to increased to $44.4 \%, 65.0 \%$ and $100.0 \%$ in the last three 5-cycle intervals. Similarly we can see that the damage percentages were lower in the first three 5-cycle intervals than the last three at 10 and 18 vibration frequency. Therefore, it might be important to reduce the continuous vibration time to achieve low fruit damage percentage, which agrees with findings in the research of utilizing intermittent harvesting technology to reduce overall fruit damage (He, Zhou, Zhang, Karkee, 2015).

Overall fruit damage percentage due to vibration was still substantially higher than the damage percentage of manually harvested fruits, which was measured as $7.5 \%$ in this study as shown by the red dash dot line in Fig. 8a. To obtain more details on damaged fruits, the overall 
367 fruit damage percentage was broken into four defined damage levels, as shown in Fig. 9. It can

368 be seen that fruits with level-1 damage (marketable fruits) account for approximately half of the

369 damaged fruit, i.e. $33.3 \%$ out of $55.2 \%$ total damaged fruits at $10 \mathrm{~Hz}, 20.6 \%$ out of $47.1 \%$ at 14

$370 \mathrm{~Hz}$, and $28.2 \%$ out of $63.4 \%$ at $18 \mathrm{~Hz}$. In higher damage level of 3 and 4 , the higher vibration

371 frequency of $18 \mathrm{~Hz}$ always resulted in the larger percentage of unmarketable fruit than $10 \mathrm{~Hz}$ and

$37214 \mathrm{~Hz}$. At each damage level, a big jump of damage percentage in the first 15 cycles was

373 evidenced in the figures, indicating the sharp increase in fruit damage. Meanwhile, the high fruit

374 damage percentage might be also contributed by the high maturity level (an overall average

375 value of $6.3 \pm 0.2$ ) due to the late harvest season, when the fruits became soft and easily

376 damaged.

377

378

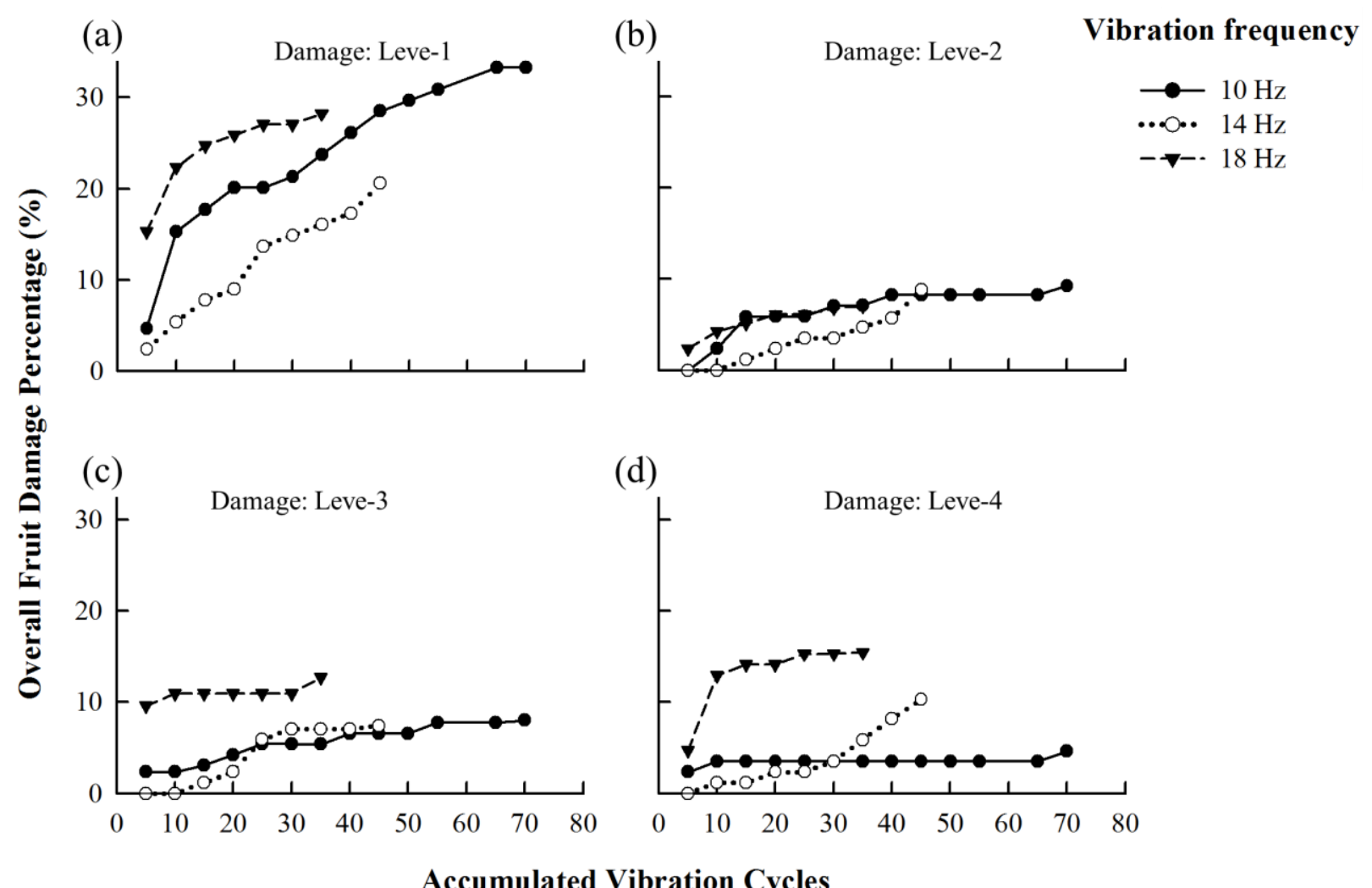

Accumulated Vibration Cycles 
Fig. 9 - The overall fruit damage percentage at different damage levels under different vibration frequencies. (a) - (d) Overall fruit damage percentage at damage level 1-4 respectively.

\subsection{Fruit impact during shaking}

The average numbers of fruit-to-fruit and fruit-to-limb impacts of all fruits are shown in

Fig. 10. The chance of fruit-to-fruit impacts was substantially higher than that of fruit-to-limb

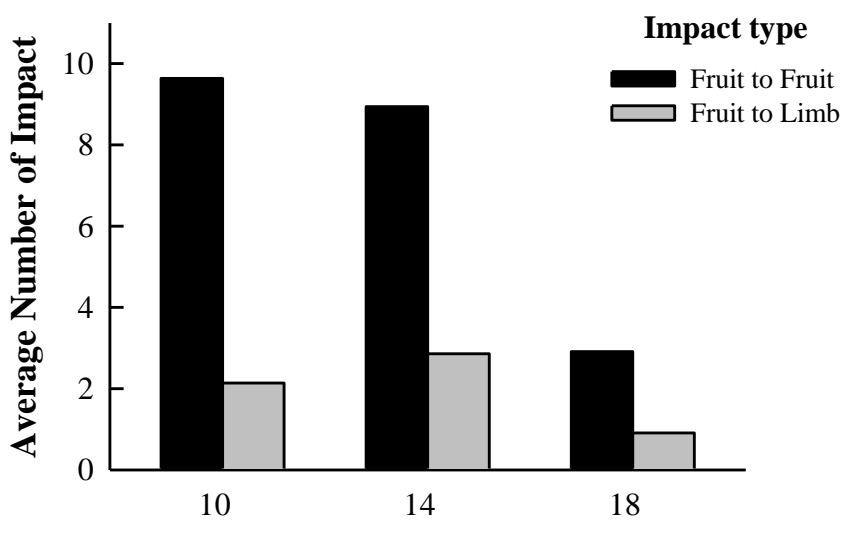

Vibration Frequency $(\mathrm{Hz})$

Fig. 10 - Average numbers of fruit-to-fruit and fruit-to-limb impact of all the test fruit at three vibration frequencies of $10 \mathrm{~Hz}, 14 \mathrm{~Hz}$ and $18 \mathrm{~Hz}$.

\section{Conclusions}

A study was conducted to investigate the cherry fruit motion patterns induced by 
401 level under corresponding conditions. The motion trajectory of tested fruits was analysed and the 402 number of cycles of four composing motion patterns under different vibration frequencies were 403 counted using high-speed images. Fruit detaching time of each fruit was also recorded. Damaged 404 fruits were classified into four levels according to the damage area. Based on the obtained results, 405 the following conclusions can be made:

406 407

(1) The motion pattern of tilting and beam-column accounted for more than $70 \%$ of all four motion patterns, indicating the importance of these two motion patterns in removal of cherry fruits.

(2) Vibration at $14 \mathrm{~Hz}$ had the lowest fruit damage percentage, mainly due to the high percentage of fruit motion in beam-column motion pattern.

(3) Lower vibration frequency resulted more low-level damage with longer detaching time, while higher frequency resulted in more high-level damage.

\section{Acknowledgments}

This research was supported in part by Washington State University Agricultural Research Center federal formula funds, Project No. WNP0745 and No. WNP0728 received from the U.S. Department of Agriculture National Institutes for Food and Agriculture (NIFA) and by the USDA Special Crop Research Initiative (SCRI) program (Project No. WPN03320). Any opinions, findings, conclusions, or recommendations expressed in this publication are those of the author(s) and do not necessarily reflect the view of the U.S. Department of Agriculture and Washington State University. The authors would also like to express their gratefulness to Mr. Patrick Scharffor his help in the fabrication of experimental equipment. 


\section{References}

Caplan, S., Tilt, B., Hoheisel, G., \& Baugher, T. A. (2014). Specialty crop growers' perspectives on adopting new technologies. HortTechnology, 24(1), 81-87.

Castro-Garcia, S., Rosa, U. A., Gliever, C. J., Smith, D., Bruns, J. K., Krueger, W. H., Glozer, K. (2009). Video evaluation of table olive damage during harvest with a canopy shaker. HortTechnology, 19(2), 260-266.

Chen, D., Du, X., Zhang, Q., Whiting, M. D., Scharf, P. A., \& Wang, S. (2012). Performance evaluation of mechanical cherry harvesters for fresh market grade fruits. Applied Engineering in Agriculture, 28(4), 483-489.

Cooke, J. R., \& Rand, R. H. (1969). Vibratory Fruit harvesting: a linear theory of fruit-stem dynamics. Journal of Agricultural Engineering Research, 14(3), 195 - 209.

Diener, R. G., Mohsenin, N. N., \& Jenks, B. L. (1965). Vibration characteristics of trellistrained apple trees with reference to fruit detachment. Transactions of the ASAE, $8(1)$, 20-24.

Du, X., Chen, D., Zhang, Q., Scharf, P. A., \& Whiting, M. D. (2012). Dynamic responses of sweet cherry trees under vibratory excitations. Biosystems Engineering, 111(3), 305314.

Halderson, J., L. (1966). Fundamental factors in mechanical cherry harvesting. Transactions of the ASAE, 9(5), 681- 684.

He, L., Zhou, J., Du, X., Chen, D., Zhang, Q., \& Karkee, M. (2013). Energy efficacy analysis of a mechanical shaker in sweet cherry harvesting. Biosystems Engineering, 116(4), 309-315. 
He, L., Zhou, J., Zhang, Q., \& Karkee, M. (2015). Evaluation of multipass mechanical harvesting on 'Skeena' sweet cherries trained to Y-trellis. HortScience, 50(8), 11781182.

Horvath, E., \& Sitkei, G. (2001). Energy consumption of selected tree shakers under different operational conditions. Journal of Agricultural Engineering Research, 80(2), 191199.

Kappel, F., Fisher-Fleming, B., \& Hogue, E. (1996). Fruit characteristics and sensory attributes of an ideal sweet cherry. HortScience, 31(3), 443-446.

Lamouria, L., \& Brewer, H. (1965). Determining selected bio-engineering properties of olives. Transactions of the ASAE, 8(3), 271 - 274.

Li, P., Lee, S.-h., \& Hsu, H.-Y. (2011). Review on fruit harvesting method for potential use of automatic fruit harvesting systems. Procedia Engineering, 23(2011), 351-366.

Li, Z., \& Thomas, C. (2014). Quantitative evaluation of mechanical damage to fresh fruits. Trends in Food Science \& Technology, 35(2), 138-150.

Norton, R. A., Claypool, L. L., Leonard, S. J., Adrian, P. A., Fridley, R. B., \& Charles, F. M. (1962). Mechanical harvest of sweet cherries. California Agriculture, 16(5), 8-10. Opara, U. L., \& Pathare, P. B. (2014). Bruise damage measurement and analysis of fresh horticultural produce-A review. Postharvest Biology and Technology, 91, 9-24.

Peterson, D. L., Whiting, M. D., \& Wolford, S. D. (2003). Fresh-market quality tree fruit harvester part I: sweet cherry. Applied Engineering in Agriculture, 19(5), 539-543. Rand, R. H., \& Cooke, J. R. (1970). Vibratory fruit harvesting: a non-linear theory of fruitstem dynamics. Journal of Agricultural Engineering Research, 15(4), 347 - 363. 
Rumsey, J. W., \& Barnes, K. K. (1970). Detachment characteristics of desert-grown oranges and grapefruit. Transactions of the ASAE, 13(4), 528-530.

Stafford, J. R., \& Diener, R. G. (1973). Design criteria for minimizing predetachment fruit damage during mechanical shaking. Transactions of the ASAE, 16(5), 840-843.

Thompson, J. F., Grant, J. A., Kupferman, E. M., \& Knutson, J. (1997). Reducing sweet cherry damage in postharvest operations. HortTechnology, 7(2), 134 - 138.

Torregrosa, A., Albert, F., Aleixos, N., Ortiz, C., \& Blasco, J. (2014). Analysis of the detachment of citrus fruits by vibration using artificial vision. Biosystems Engineering, 119(2014): 1-12.

USDA-AMS. (1997). United States Standards for Grades of Sweet Cherries. Agricultural Marketing Service. Washington, D.C.: USDA Agricultural Marketing Service. Available at: www.nass.usda.gov. Accessed 23 August 2013.

USDA-AMS. (2005). Sweet Cherries: Shipping Point and Market Inspection Instructions. Agricultural Marketing Service. Washington, D.C.: USDA Agricultural Marketing Service. Available at: www.nass.usda.gov. Accessed 23 August 2013.

Zhou, J., He, L., karkee, M., \& Zhang, Q. (2013). The effect of shaking frequency on fruit motion patterns and damage rate during cherry detaching process. Paper presented at the 2013 ASABE Annual International Meeting, Kansas City, Missouri.

Zhou, J., He, L., Karkee, M, \& Zhang, Q. (2016). Effect of catching surface and tilt angle on bruise damage of sweet cherry due to mechanical impact. Computers and Electronics in Agriculture, 121(2016), 282-289. 
Zhou, J., He, L., Zhang, Q., Du, X., Chen, D., \& Karkee, M. (2013). Evaluation of the influence of shaking frequency and duration in mechanical harvest of sweet cherry. Applied Engineering in Agriculture, 29(5), 607-612.

Zhou, J., He, L., Zhang, Q., \& Karkee, M. (2014). Effect of excitation position of a handheld shaker on fruit removal efficiency and damage in mechanical harvesting of sweet cherry. Biosystems Engineering, 125(2014), 36-44. 